\title{
GUEST EDITORIAL Special Issue: Geometric representation and reasoning in design
}

\author{
ROBERT WOODBURY AND CARLOS DAMSKI \\ Faculty of Architecture, Landscape Architecture and Urban Design, The University of Adelaide, South Australia 5005, Australia
}

This special issue is devoted to the role of geometric representation and reasoning in computational models of design. On one hand, representations establish relations between concepts to be modelled and the symbol system that represents them. On the other, reasoning describes the high-level operations that are defined over a symbol system and the effects such operations have on the concepts being modelled. Representation may be taken as encompassing computation and thus reasoning - the argument goes that a symbol system is only fully specified when the syntax and semantics of all of its symbols, relations, and algorithms are known. We have taken the license of identifying reasoning as a distinct part of the enterprise to emphasize a correspondence between computational processes and the design work they support. It is our hope that the computational processes reported in this special issue bear a direct relation, a domain proximity, to the design tasks they are intended to model, support, and perhaps transform.

Why then our focus on geometry? Certainly geometry is a major aspect of the concepts for which we build computational representations, but it is not the only one. It does, however, invoke difficulties that make it an appropriate focus for research in computational design. Geometric concepts exist in multidimensional spaces, some continuous, others discrete, and, in a given model, these dimensions usually interact. It is precisely such interactions that bedevil the quest for good representations and have made the field such a fertile yet sometimes unyielding ground for research.

In this special issue we have gathered together a set of seven papers that address diverse facets of geometric computation in design. Each addresses an aspect of design. Each aims to model, support, and possibly transform this aspect in a particular way. Each has its own model, representation, and processes of reasoning.

Reprint requests to: Dr. Robert Woodbury, Faculty of Architecture, Landscape Architecture and Urban Design, The University of Adelaide, South Australia 5005, Australia. Telephone: +6188303 4590; Fax: +6188303 4377; E-mail: rw@arch.adelaide.edu.au
The first paper, Spatial Synthesis by Disjunctive Constraint Satisfaction by C. Baykan and M. Fox, addresses the problem of synthesis of spatial layouts, which is an issue in fields ranging from circuit layout to building design. Baykan and Fox make the common simplifying assumption that the objects of interest can be approximated as rectangles and that such occur arranged in 2D continuous space. Twodimensional arrangements of rectangles have been the focus of many research efforts: what distinguishes the present work is its direct treatment of these as disjunctive constraints over real variables. A selection of one disjunct from each of a set of disjunctive constraints defines an equivalence class of layouts each member of which is essentially the same with respect to its domain. Disjunctive constraints are formally a disjunctive constraint satisfaction problem, which is known to be NP-complete. Thus, heuristics are needed for effective computation and these take the form of heuristics on the order in which disjunctive constraints are considered in a process.

The second paper, Algorithms for Computing Intersections and Union of Toleranced Polygons with Applications by F. Cazals and G. Ramkumar, dives into the relations that can exist between instances of toleranced polygons. They develop a representation for toleranced polygons and efficient algorithms for computing intersection and union of such. At the end of the paper, they discuss possible applications for their results: these range from analysis of mechanisms the behavior of which depends on interference in the assembly process; through determination of the critical parameters of failed assembly sequences; to detailed analysis of critical points in robotic motion.

The third paper, Modelling Spatial Reasoning Systems with Shape Algebras and Formal Logic by S. Chase, presents a blend of two paradigms to represent shape and spatial relations: shape algebra and predicate logic. In essence, relations in a shape algebra are taken as fundamental and logic is used to combine these into higher order relations. The result has advantages in describing shapes and spatial relations in domains such as architecture and geographic in- 
formation systems. Implementation is a long-standing concern in shape grammar research, which this paper, like most in the area, does not address directly, though it acknowledges the difficulties in and promising directions for future research.

The fourth paper, Prototype System for Supporting the Incremental Modelling of Vague Geometric Configurations by X. Guan, A.H.B. Duffy, and K.J. MacCallum, addresses what the authors call vagueness, the reality that designers deal with abstractions, in which aspects of a design may be specified to varying degrees of completeness as design proceeds. They respond to this well-known and difficult research issue by developing a prototype system based on constraints, minimum commitment, and incremental refinement. Their system is object-based, that is, the objects to be modelled and the constraints on them are represented as collections of data objects. Computing over this representation is accomplished by incremental addition or retraction of constraints, resolution of which constraints apply, formulation of an instance of a constraint problem and solution of that constraint problem by a general purpose solver. At the end of the paper, the authors correctly identify that human computer interfaces issues are inter alia important to the productive application of this work.

The fifth paper, Qualitative Sketch Optimization by A. Mukerjee, R. Agrawal, N. Tiwari and N. Hasan, begins with ambiguity, the oft-observed phenomenon in designing that representations stand for classes of objects rather than specific objects. Representations for early design are thus abstractions, each of which denotes a set of more specific designs. The authors argue through several of the published approaches to abstraction, especially those falling into the wide area of qualitative reasoning. They then offer their own approach, a parameterized Medial Axis Transform (MAT) which they call a QMAT. Inverting the usual application of the MAT, which is to determine features from an object's form and is known to be highly unstable, they use the MAT directly as an abstract representation of a form. This makes their representation stable in the sense that closely related instances of a MAT result in closely related instances of the represented forms.

The sixth paper, Representing and Reasoning about Geometric Tolerances in Design by J. Tsai and M. Cutkosky, presents a tolerancing representation schema, called the Tolerance Network, which is a generic model to represent Geometric Dimensioning and Tolerancing (GD\&T) specifications. The paper also presents a comprehensive literature review of work in GD\&T in CAD.

The final paper, Solving Regional Constraints in Components Layout Design based on Geometric Gadgets by $\mathrm{H}$. Suzuki, T. Ito, H. Ando, K. Kikkawa and F. Kimura, discusses a new method for dealing with geometrical layout constraints of three types: dimensional, regional, and interference. The method converts regional, and interference constraints into a set of simple inequalities that are solved by a "geometric gadget," which is a structured set of dimensional constraints. A prototype system is presented with some layout design examples.

We believe that the papers on this special issue represent, and give a significant contribution to, a wide variety of types of representation and reasoning using models of geometry in design problems. 